\title{
Austria-based real-world data on bevacizumab in newly diagnosed epithelial ovarian cancer
}

\author{
Irina Tsibulak $(\mathbb{D}) \cdot$ Stephan Polterauer · Alexander Reinthaller · Christian Schauer · Jürg Berger · Christian Marth
}

Received: 14 October 2021 / Accepted: 11 January 2022 / Published online: 10 February 2022

(C) The Author(s) 2022

\begin{abstract}
Summary
Background Front-line maintenance therapy with bevacizumab demonstrates high efficacy and safety in epithelial ovarian cancer, as already shown in large phase III trials; however, the corresponding study populations are often not fully representative of patients in clinical routine. In this Austria-based multicenter study, we aimed to explore the real-world outcomes of bevacizumab use in front-line treatment of ovarian cancer, including patients with comorbidities and poor performance status.

Patients This study is an open label single arm multicenter noninterventional trial and included patients with newly diagnosed advanced epithelial ovarian cancer, who were treated with platinum-based chemotherapy and were candidates for receiving bevacizumab according to the product label. Data
\end{abstract}

Supplementary Information The online version of this article (https://doi.org/10.1007/s00508-022-02005-2) contains supplementary material, which is available to authorized users.

\section{Tsibulak · C. Marth (价}

Department of Obstetrics and Gynecology, Medical

University Innsbruck, Anichstraße 35, 6020 Innsbruck, Austria

christian.marth@tirol-kliniken.at

S. Polterauer · A. Reinthaller

Department of Obstetrics and Gynecology, Comprehensive Cancer Center Vienna, Medical University Vienna,

Währinger Gürtel 18-20, 1090 Vienna, Austria

\section{Schauer}

Department of Gynecology, Konventhospital Barmherzige

Brueder, Marschallgasse 12, 8020 Graz, Austria

\section{J. Berger}

Roche Austria GmbH, Engelhorngasse 3, 1210 Vienna, Austria collection started in the third quarter of 2012 and ended in the third quarter of 2018.

Results In this study 50 patients were included and 575 adverse events were reported for $90 \%$ of the patients. The majority of the adverse events were mild (47\%) or moderate (37\%). The most common adverse events were hypertension $(60 \%)$, anemia $(48 \%)$, leukopenia (42\%), thrombocytopenia (36\%), neutropenia (36\%) and proteinuria (26\%). A relation to bevacizumab was documented only for $10.3 \%$ of all adverse events. In almost $50 \%$ of all adverse events, no intervention was needed and bevacizumab treatment had to be interrupted only in $3.3 \%$ of all adverse events. The median progression-free survival was 1.3 years $(95 \%$ CI 1.1-1.8).

Conclusion The routine use of front-line bevacizumab for advanced ovarian cancer is associated with high efficacy comparable with that obtained in randomized phase III clinical trials; however, hypertension and proteinuria were reported significantly more often in our Austria-based real-world population.

Keywords Ovarian carcinoma - Survival · Adverse events · Anti-angiogenesis

\section{Introduction}

Epithelial ovarian cancer (EOC) is the seventh most common cancer among women in Austria and belongs to the leading causes of cancer death in females in the western world [1]. In 2018, 761 women were diagnosed with EOC in Austria and 520 of them succumbed to this malignant disease [1]. EOC is most likely to be diagnosed at advanced stages, requiring extensive surgical cytoreduction as well as platinumbased systemic treatment [2]. During the last two decades, targeted therapies and the concept of maintenance therapy became increasingly more important 
in the management of EOC. Angiogenesis plays a crucial role in ovarian cancer progression and therefore represents an essential therapeutic target in treatment of EOC. During the last years, several antiangiogenic agents have been investigated in EOC and of these only bevacizumab, a monoclonal antibody targeting vascular endothelial growth factor, has been introduced in the treatment of ovarian cancer. Based on the results of ICON-7 and GOG0218 trials, frontline maintenance therapy with the angiogenesis inhibitor bevacizumab was approved in Europe and since 2018 it has also been approved in the USA [3, 4]. The most common adverse events of this agent are hypertension and proteinuria; however, these rarely lead to bevacizumab discontinuation [3, 4]. At the present time, front line maintenance therapy with bevacizumab is broadly used and demonstrates high efficacy and safety, as already shown in large phase III trials [3-5].

It is without any doubt that these trials provide excellent evidence of efficacy; however, the corresponding study populations are often not fully representative of patients in clinical routine, as prospective clinical trials tend to have strict eligibility criteria. In this Austria-based noninterventional multicenter study, we aimed to explore the real-world outcomes of bevacizumab use in front-line treatment of EOC, including patients with comorbidities and poor performance status.

\section{Patients, material and methods}

The present study was designed as an open label single arm multicenter non-interventional trial (sponsored by Hoffmann-La Roche under ML28355; ClinicalTrials.gov number NCT01788995). The participating centers included gynecological departments at the Medical University of Innsbruck, Medical University of Vienna, and Konventhospital Barmherzige Brüder in Graz. The ethics committee of the Medical University of Innsbruck, Austria approved the present study (IRB approval\#: UN4727_NIS; 21.06.2012).

Patients aged $\geq 18$ years with newly diagnosed advanced EOC, who were treated with platinum-based chemotherapy and were candidates for receiving bevacizumab according to the product label, were eligible for the study. Patients received bevacizumab $15 \mathrm{mg} / \mathrm{kg}$ body weight every 3 weeks combined with standard front-line therapy with carboplatin and paclitaxel and then continued as single agent maintenance therapy. The inclusion of patients was based on the physician's decision. There were no specific exclusion criteria as long as patients received bevacizumab according to the drug approval.

In this trial 60 patients were planned to be included. Written informed consent was obtained for every patient. Clinical pathological characteristics were collected using an electronic Case Report Form (eCRF). Data collection started in the third quarter of 2012 and ended in the third quarter of 2018. Descriptive statistics including the occurrence in total and in percent, the minimum and maximum values, the median and the arithmetic means, were performed. No hypothesis was predefined regarding the primary and secondary objectives of the study. Progression-free survival was defined as the interval between first dose of front-line therapy until documented disease progression. Missing data were replaced with "NA" (not applicable).

\section{Results}

Due to sponsor's decision, the recruitment was stopped as 57 patients with newly diagnosed advanced EOC were recruited in this study. Of the patients 7 were excluded from the analysis as they did not receive a first-line platinum-based chemotherapy in combination with bevacizumab, so that 50 patients who received systemic treatment with carboplatin/ paclitaxel and bevacizumab were included in the analysis. Bevacizumab starting dose was $15 \mathrm{mg} / \mathrm{kg}$ body weight every 3 weeks for all patients. The median number of bevacizumab cycles was 15 cycles (range 1-30 cycles). Treatment interruptions caused by adverse effects or planned surgical procedures were reported for $33(66 \%)$ patients. The median treatment duration was 1.0 years (range $0.1-1.7$ years) and the median follow up time was 2.25 years (range $0.42-4.92$ years).

The median age of the patients was 60 years, while almost $80 \%$ were 50 years and older. Most of the patients $(78 \%)$ presented with Eastern Cooperative Oncology Group (ECOG) performance status 0 and $22 \%$ with ECOG status 1 (Supplementary Table 1).

Of the patients $64 \%$ presented with Fédération Internationale de Gynécologie et d'Obstétrique (FIGO)

Table 1 Most common adverse events per patient

\begin{tabular}{|l|l|l|}
\hline Adverse Event & $n$ Patients & $\%$ Patients \\
\hline Hypertension & 30 & 60.0 \\
\hline Anemia & 24 & 48.0 \\
\hline Leukopenia & 21 & 42.0 \\
\hline Neutropenia & 18 & 36.0 \\
\hline Thrombocytopenia & 18 & 36.0 \\
\hline Proteinuria & 13 & 26.0 \\
\hline Urinary tract infection & 13 & 26.0 \\
\hline Drug hypersensitivity & 11 & 22.0 \\
\hline Nausea & 9 & 18.0 \\
\hline Infection & 6 & 12.0 \\
\hline Diarrhea & 5 & 10.0 \\
\hline Fatigue & 5 & 10.0 \\
\hline Vomiting & 5 & 10.0 \\
\hline Constipation & 4 & 8.0 \\
\hline Pain in extremity & 4 & 8.0 \\
\hline General physical health deterioration & 4 & 8.0 \\
\hline Thrombocytosis & 4 & 8.0 \\
\hline Urinary tract infection bacterial & 4 & 8.0 \\
\hline
\end{tabular}


stage IIIc disease, $18 \%$ with FIGO stage IIIb and 14\% had a stage IV disease. The detailed histology was not routinely documented in each center; however, the cohort of 29 patients at the Department of Obstetrics and Gynecology of the Medical University of Innsbruck consisted of 1 patient with endometrioid ovarian carcinoma, 1 patient with low grade serous ovarian cancer and 27 patients with high grade serous carcinoma.

More than $55 \%(n=28)$ of patients had no residual tumor after cytoreductive surgery, while in $30 \%$ $(n=15)$ the complete resection was not achieved and in $14 \%(n=7)$ residual disease was not documented.

For $24 \%(n=12)$ of patients pre-existing hypertension was documented. The summary of pre-existing comorbidities is presented in Supplementary Table 2 and 20 patients $(40 \%)$ had no pre-existing comorbidities.

Overall, 575 adverse events (AEs) were reported for $45(90 \%)$ of the 50 patients. The majority of the reported AEs were mild ( $n=268,47 \%)$ or moderate $(n=215,37 \%)$ and 92 severe AEs were documented (16\%). The most common AEs were hypertension occurring in $60 \%$ of patients $(n=30)$, anemia ( $n=24$, $48 \%)$, leukopenia ( $n=21,42 \%)$, thrombocytopenia ( $n=18,36 \%)$, neutropenia $(n=18,36 \%)$ and proteinuria ( $n=13,26 \%$ ) (Table 1$)$; however, a relation to bevacizumab was documented only for $10.3 \%$ of all AEs $(n=59)$, while $33.4 \%(n=192)$ were related to carboplatin and $38.3 \%(n=220)$ were related to paclitaxel.

In almost $50 \%$ of all AEs, no intervention was needed, $35.5 \%$ of AEs were treated with medication therapy and in $3.3 \%(n=23)$ of all AEs bevacizumab therapy had to be interrupted. Of these $3.3 \%$, only $1 \%(n=7)$ of AEs were associated with bevacizumab (proteinuria in $n=3$ and hypertension in $n=4$ ).

Progressive disease was documented for $36(72 \%)$ patients, while median progression-free survival (PFS) was 1.3 years (95\% CI 1.1-1.8) (Fig. 1). Death occurred in $11(22 \%)$ patients, no median overall survival (OS) could be calculated (not reached).

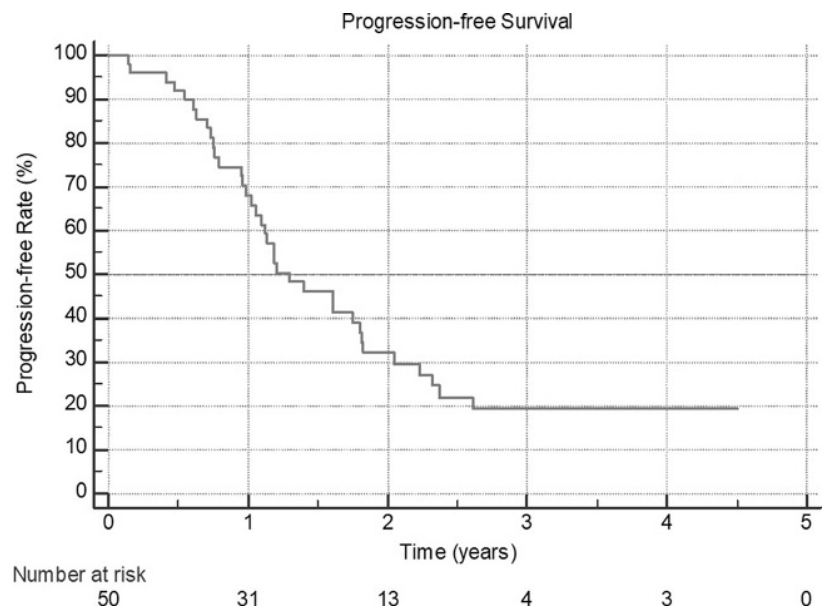

Fig. 1 Kaplan-Meier curve for progression-free survival

\section{Discussion}

In this multicenter noninterventional study of bevacizumab in newly diagnosed ovarian cancer we evaluated the routine clinical use in three Austrian centers. The combination of bevacizumab and carboplatin/ paclitaxel was well tolerated, as already shown in the large phase III trials (GOG0218, ICON7) [3, 4]. In accordance with these trials, most adverse events were typical of the chemotherapy regimen (such as anemia, neutropenia, thrombocytopenia) and were not related to bevacizumab; however, in our population, hypertension and proteinuria were reported significantly more often: hypertension in $60 \%$ in our study vs. $26 \%$ in ICON7 and $23 \%$ in GOG0218; proteinuria in $26 \%$ vs. $5 \%$ and $1.6 \%$, respectively $[3,4]$. Similar results were showed for hypertension in the single arm ROSiA trial ( $55 \%$ of patients), but still much lower incidence of proteinuria (7.6\% of patients) was documented in ROSiA as compared to our study [5]. This high incidence of hypertension as an adverse event could be due to the fact that $24 \%$ of our patients already had a pre-existing hypertension. We assume that the high incidence of proteinuria is not due to comorbidities, as no pre-existing proteinuria was reported before bevacizumab in our cohort; however, proteinuria was reported for $20 \%$ of patients in OSCAR study, which was a UK-based observational study of front-line bevacizumab in advanced EOC [6]. In our study, no gastrointestinal perforation and no wound healing complications occurred, probably due to the small population size, as these complications had a low incidence rate in larger trials: gastrointestinal perforation in $>1 \%$ of patients in ICON7, $2.6 \%$ in GOG0128 and 2\% in the observational OSCAR study; wound healing complication in $5 \%, 3 \%$ and $2 \%$ of patients, respectively $[3,4,6]$. Approximately $80 \%$ of AEs in our cohort were resolved or showed improvement and bevacizumab had to be interrupted in only $3.3 \%$, so that no new safety signals emerged.

The median time to progression in our population was 15.6 months, which is comparable to the GOG218, ICON7 and OSCAR study results $(14.1,16.0$ and 15.4 months, respectively) $[3,4,6]$. On the other hand, the PFS of 15.6 months in our study is significantly shorter than PFS of 25.5 months in the international ROSiA study [5]; however, in ROSiA bevacizumab treatment was continued up to 24 months and $23 \%$ of patients had a stage I-IIIA disease, whereas the median number of bevacizumab cycles in our study was 15 cycles and $96 \%$ of our patients presented with FIGO stage IIIb-IV disease.

The main limitations of our study are the small patient size and the lack of a control arm.

To our knowledge, this is the first study which reflects real-world Austria-based clinical practice, including patients with pre-existing morbidities. Our results show encouraging evidence that the routine use of front-line bevacizumab for advanced ovarian 
cancer is associated with high safety and efficacy comparable with those obtained in randomized phase III clinical trials; however, hypertension and proteinuria were reported significantly more often in our Austriabased real-world population.

Funding Open access funding provided by University of Innsbruck and Medical University of Innsbruck.

Conflict of interest I. Tsibulak received honoraria for lectures and congress travel support from Roche (not related to this manuscript). S. Polterauer received honoraria for lectures from Roche (not related to this manuscript). A. Reinthaller declares no competing interests. C. Schauer declares no competing interests. J. Berger receives salary as employee of Roche Austria. C. Marth received honoraria for lectures, congress travel support and consulting fees from Roche, he also declared participation on a data safety monitoring board and advisory board for Roche (not related to this manuscript).

Open Access This article is licensed under a Creative Commons Attribution 4.0 International License, which permits use, sharing, adaptation, distribution and reproduction in any medium or format, as long as you give appropriate credit to the original author(s) and the source, provide a link to the Creative Commons licence, and indicate if changes were made. The images or other third party material in this article are included in the article's Creative Commons licence, unless indicated otherwise in a credit line to the material. If material is not included in the article's Creative Commons licence and your intended use is not permitted by statutory regulation or exceeds the permitted use, you will need to obtain permission directly from the copyright holder. To view a copy of this licence, visit http://creativecommons.org/licenses/by/4.0/.

\section{References}

1. Statistics Austria. Eierstock. 2021. http://www.statistik.at/ web_de/statistiken/menschen_und_gesellschaft/gesund heit/krebserkrankungen/eierstock/index.html. Accessed 10 June 2021.

2. Tsibulak I, Zeimet AG, Marth C. Hopes and failures in frontline ovarian cancer therapy. Crit Rev Oncol Hematol. 2019;143:14-9.

3. Perren TJ, Swart AM, Pfisterer J, et al. A phase 3 trial of bevacizumab in ovarian cancer. NEnglJ Med. 2011;365:2484-96.

4. Burger RA, Brady MF, Bookman MA, et al. Incorporation of bevacizumab in the primary treatment of ovarian cancer. NEngl J Med. 2011;365:2473-83.

5. Oza AM, Selle F, Davidenko I, et al. Efficacy and safety of bevacizumab-containing therapy in newly diagnosed ovarian cancer: ROsiA single-arm phase 3B study. Int J GynecolCancer. 2017;27:50-8.

6. Hall M, Bertelli G, Li L, et al. Role of front-line bevacizumab in advanced ovarian cancer: the OSCAR study. Int J Gynecol Cancer. 2020;30:213-20.

Publisher's Note Springer Nature remains neutral with regard to jurisdictional claims in published maps and institutional affiliations. 